\title{
A Design for Portable Camera Fixture with Multiple Degrees of Freedom
}

\author{
LuQian Duan ${ }^{1}$, XueGuo $\mathrm{An}^{2}$, JunWen Xing ${ }^{1}$ \\ ${ }^{1}$ Department of Mechanical Engineering, Academy of Armored Forces Engineering, Beijing 100072, \\ China \\ ${ }^{2}$ Foreign Training Department, Academy of Armored Forces Engineering, Beijing 100072, China \\ Email: mary.duan@aliyun.com
}

Keywords: Multiple degrees of freedom; Camera fixture; Design

Abstract: A portable and adjustable camera fixture with multiple degrees of freedom is designed so as to solve the problem of fixing and adjusting the camera used in the trial of the tank equipped with newly-developed gas turbine. It is simple in structure, flexible and easy in use, and secure and reliable in fixing. With the help of the fixture, the camera can be adjusted easily and rapidly to any required angle, getting an ideal shoot angle. It is a perfect company of the steel vehicles and equipment like car, tank, armored vehicle, ship, and so on.

\section{Introduction}

Because of the demand for bigger traction, most of tanks in the world prefer to use the diesel engine with high power density. But on plateau, oxygen deficit may cause the frequent shutdown of the engine as its load is increased, limiting the normal performance of the engine.

Compared with the diesel engine, the gas turbine has some advantages, such as quicker start, higher power density, simpler structure, and lower additional power loss, and so on. It can use diesel as the fuel as well. But the high-density oxygen supplied by compressed air can assist the combustion, which has effectively solved the problem of the shutdown of the engine when working on plateau.

China has a large power in developing and manufacturing tank. The vast in territory and variety of climate and terrain give rise to the tactical demand for developing and manufacturing the tank gas turbine. The sustainable development of the gas turbine technology has laid a solid technical foundation for developing the gas turbine equipped in the tank. Chinese researchers have developed the first sample tank equipped with a gas turbine after years of unremitting efforts, and done successfully various tests including the plateau test, greatly improving the stability of power system of tank on plateau.

In order to analyze the performance index of the engine, it is necessary to record by video the indications of all the instruments in the driver's compartment during the whole process of test. The video camera generally needs to be fixed when working in running car, tank, armored vehicle and ship. But it is difficult to get an ideal shooting angle if the camera is fixed by the current fastening means. What is more, the camera cannot be adjusted quickly and flexibly after it is fixed, bringing about the difficulty in the use of the camera.

In this article, a design of a portable and adjustable camera fixture with multiple degrees of freedom is introduced. It is easy for adjusting and fixing a camera, effectively solving the problem of fixing and adjusting the camera in the trial of the tank equipped with gas turbine. 


\section{The Design of Camera Fixing}

Application Environment. As we know, the driver's compartment of a tank is very small in space, allowing only one person sitting there. Its inner wall is made of ferrous metallic material. The ceiling is rough and curve. The instrument panel is arranged on the left side of the driver. So, the camera for recording the readings of the instruments has to be fixed on the floor, ceiling or opposite sidewall of the compartment.

If fixed on the sidewall, however, it will be blocked by the driver. If fixed on the floor, it must be supported by a stand like tripod, but the compartment is not big enough to arrange such a stand. As a result, the only possible position for fixing the camera is the ceiling of the compartment.

The Design of Fixing Means. How to fix the camera on the ceiling of the driver's compartment?

Two means can be adopted. One is destructive, another non-destructive.

The camera can be fixed on the ceiling of the compartment by means of drilling, threading or welding. Its position is expected to be different as the height of the driver may be different. Otherwise, the camera may be blocked by the driver, causing the incomplete recording of the data. Furthermore, fixing the camera by such means will damage the inner structure of the compartment. So it is inadvisable.

Sticking or adsorption can be used as the non-destructive fixing means. The former means to stick the camera on the ceiling of the driver's compartment by glue. But once the camera is fixed by glue, it cannot be moved any more unless re-stuck. So it is not advisable. The latter, however, is to fix the camera by means of vac-sorb. But it requires the ceiling surface of the compartment must be as smooth as a mirror. Otherwise, the camera is unable to be fixed if there is air existing between the roof and the camera. So the means of vac-sorb is not acceptable either as the surface of the ceiling is rough.

However, a magnet can be adsorbed onto the inner wall of the compartment as it is made of ferrous metallic material. Another adsorption means can be considered, magnetic adsorption, which means the camera can be fixed onto the compartment ceiling by an easily-mounted magnet. The fastening is reliable and non-destructive to other parts. So the magnetic means is selected finally for fixing the camera.

Select a magnet with proper size and enough magnetic force according to the size and weight of the camera. In the design, as the camera is of mini type, it can be fixed firmly to the ceiling of the compartment by a magnet with the section area of a One-Yuan coin and the height of $20 \mathrm{~mm}$. The fixing is proved to be firm and reliable even when the tank runs on the irregular ground. In addition, the camera can be fixed on the ceiling or taken off at any moment as needed. What is more, the camera can be fixed easily at any proper position in accordance with the height of different drivers and has no any damages on the inner structure of the compartment.

\section{The Design of Adjusting Means for the Camera Fixture}

Structure Design of Fixture. The camera can be fixed on the compartment ceiling by a magnet. But as the ceiling is a curved surface instead of a smooth plane, it is necessary to design a device with the functions of fixing and adjusting at the same time so as to obtain an ideal shoot angle. That specially designed device is called camera fixture.

The sketch of the camera fixture is shown in Fig.1. It is composed of an adsorption block 1 made of magnet, a connecting rod with thread 2, an L-shaped bracket and a mounting seat. The adsorption block may be cylindrical or cubic. The L-shaped bracket consists of two parts: a horizontal plate 3 and a vertical plate 5, and both of them have a through hole in the center. 


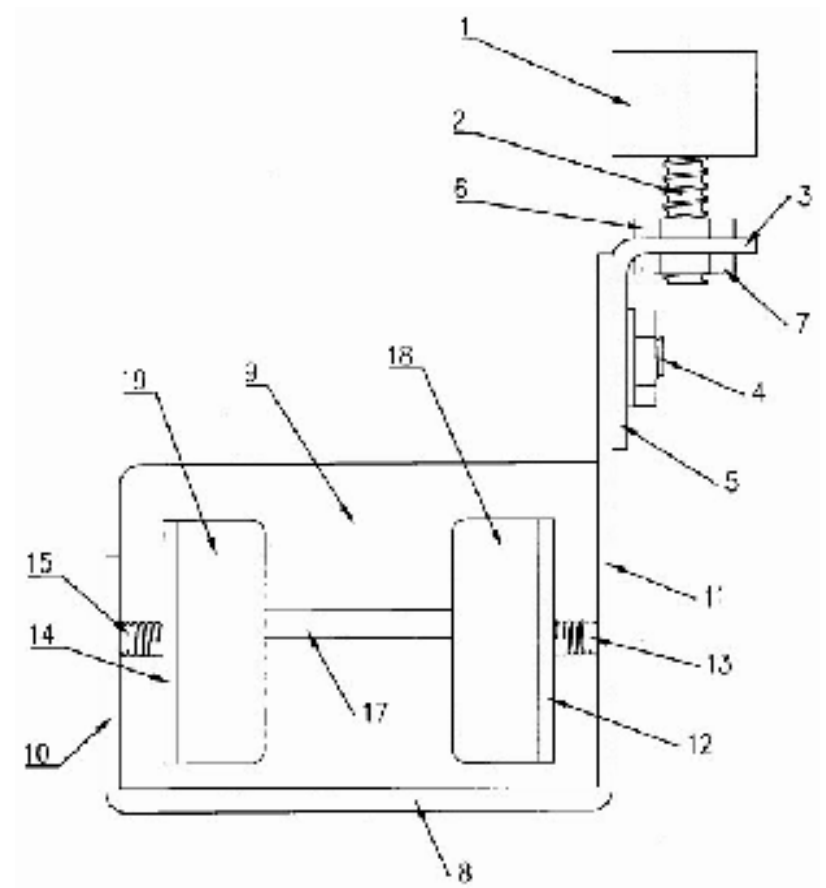

a. The Front View of the Camera Fixture

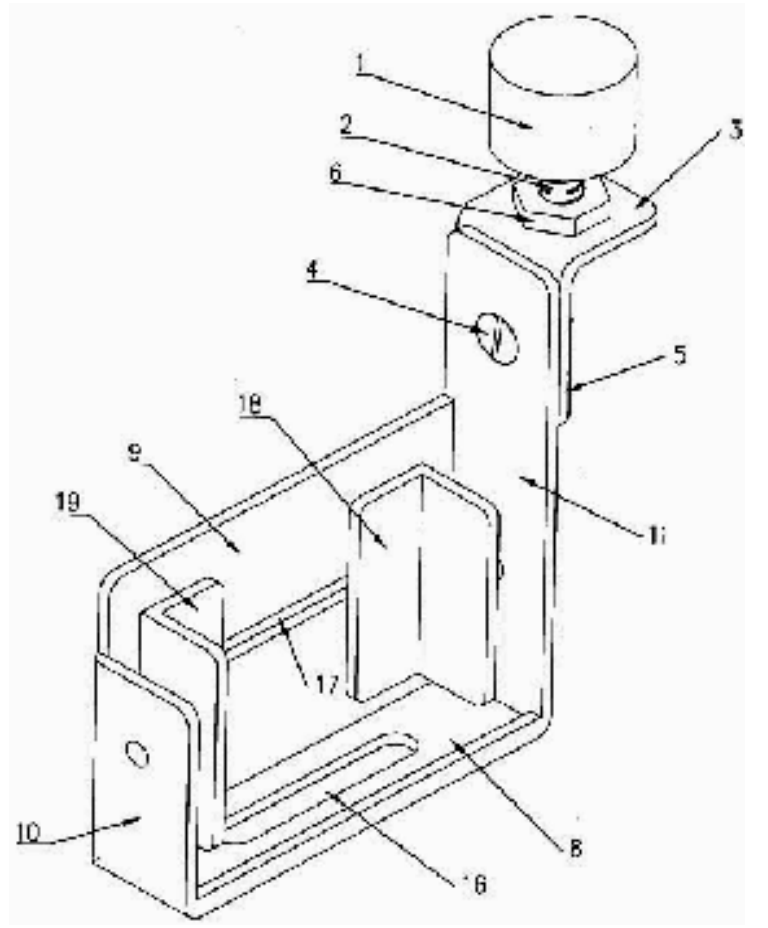

b. The Axonometric View of the Camera Fixture

1. Adsorption Block 2.Connecting Rod 3.Horizontal Plate 4.Adjusting Screw 5. Vertical Plate 6. Upper Nut 7. Lower Nut 8.Bottom Plate 9.Back Plate 10.Left Side Plate 11.Right Side Plate 12.Right Stop Plate 13.Right Spring 14.Left Stop Plate 15.Right Spring 16. Slot on Bottom Plate 17. Slot on Back Plate 18.Right Guard Plate 19. Left Guard Plate

Fig. 1 The Sketch of the Camera Fixture

One end of the connecting rod 2 is joined by threads to the adsorption block, while the other end connected with the horizontal plate 3 of the L-shaped bracket. The vertical plate 5 of the L-shaped bracket is connected with the camera mounting seat through an adjusting screw 4 whose 
axis is perpendicular to that of the connecting rod 2.

The camera mounting seat is made up of a rectangular bottom plate 8 , a back plate 9 , and left and right side plates 10 and 11, which are perpendicular to each other as shown in Fig.1b. Two long and through slots 16 and 17 from left to right are cut respectively on the bottom and the back plates for housing the connecting wires of the camera.

Two L-shaped movable stop seats are provided on the bottom plate 8, consisting of the left and right stop plates 12 and14, and the left and the right guard plates 18 and 19. The stop plates, being perpendicular to the guard plates, are connected with the two side plates through the left and right springs 15 and 13. This kind of design can fix the camera firmly and be suitable for cameras with different dimensions.

Application of the fixture. Install the camera in between the two movable seats before the trial of the tank equipped with gas turbine. Keep the back of the camera pressed against the two guard plates tightly while its two sides against the two side plates tightly. Clamp the camera tightly by the two springs. Then, fix the camera fixture at a proper position on the ceiling of the driver's compartment by the adsorption block 1. Adjust the upper nut 6 and lower nut 7, as shown in Fig. 1a, to move and rotate the L-shaped bracket along the axis of the connection rod 2 so as to locate its fixing position. Lastly, adjust the position of the camera mounting seat through the adjusting screw 4 to provide the camera with an ideal shoot angle.

It is observed from the above that the adsorption block 1 ensures the flexible movement and rotation of whole camera fixture on the compartment ceiling. The connecting rod 2 with threads on the fixture realizes the up and down movement of the camera mounting seat together with the L-shaped bracket. The adjusting screw 4 on the fixture enables the camera mounting seat to rotate around the axis of the connecting rod 2. The above actions provide the camera with five degrees of freedom including two rotations around the coordinates $\mathrm{X}$ and $\mathrm{Y}$, and three displacements along the coordinates $\mathrm{X}, \mathrm{Y}$ and $\mathrm{Z}$ in space coordinate system. So the camera fixture can be adjusted flexibly. It can be seen from Fig. 1 that the design is simple in structure and convenient to carry, meeting all the demands of use.

\section{Conclusions}

In this article, a design of a portable and adjustable camera fixture with multiple degrees of freedom is introduced. Before use, first fasten the fixture on the surface of the part made of the ferrous metallic material with the adsorption block made of permanent magnet. Then, adjust the position of the L-shaped bracket along the connecting rod axis and its rotating direction, and move the camera mounting seat to the proper position through the adjusting screw to provide the camera in the mounting seat with an ideal shoot angle. So, it is simple in structure, flexible and easy in use, and secure and reliable in fixing. With the help of the fixture, the camera can be adjusted easily and rapidly to any required angle, getting an ideal shoot angle. It is a perfect company of the steel vehicles and equipment like car, tank, armored vehicle, ship, and so on.

The design has effectively solved the problem of fixing and adjusting the camera in the trial of the tank equipped with gas turbine. It is also instructive to settle the fixing and adjusting of any device on the surface of the ferrous metallic material. 


\section{Reference}

[1] TV Blalock, EJ Kennedy, VA Thomason. Development of a digital electronic rebalance loop for a dry tuned-rotor two degree-of-freedom gyroscope, Nasa Sti/recon Technical Report N1975

[2] ZM Ge, C.L. Hsiao, Y.S. Chen. Chaos and Chaos Control for a Two-Degree-of-Freedom Heavy Symmetric Gyroscope : International Journal of Nonlinear Sciences and Numerical Simulation, - 2011 